\title{
CHARACTERIZING MICROFLUIDIC OPERATIONS UNDERLYING AN ELECTROWETTING HEAT PIPE ON THE INTERNATIONAL SPACE STATION
}

\author{
Enakshi Wikramanayake* \\ Department of Mechanical Engineering \\ The University of Texas at Austin \\ Austin, Texas, USA
}

\author{
Renee Hale \\ McKetta Department of Chemical \\ Engineering \\ The University of Texas at Austin \\ Austin, Texas, USA
}

\author{
John Elam \\ Department of Mechanical Engineering \\ The University of Texas at Austin \\ Austin, Texas, USA
}

\author{
Arjang Shahriari \\ Department of Mechanical Engineering \\ The University of Texas at Austin \\ Austin, Texas, USA \\ Angel R. Alvarez-Hernandez \\ NASA Johnson Space Center \\ Houston, TX, USA
}

\author{
Vaibhav Bahadur \\ Department of Mechanical Engineering \\ The University of Texas at Austin \\ Austin, Texas, USA \\ Nathan Howard \\ NASA Johnson Space Center \\ Houston, TX, USA
}

\section{ABSTRACT}

Electrowetting heat pipes (EHPs) are a newly conceptualized class of heat pipes, wherein the adiabatic wick section is replaced by electrowetting-based pumping of the condensate (as droplets) to the evaporator. Specific advantages include the ability to transport high heat loads over long distances, low thermal resistance and power consumption, and the absence of moving mechanical parts. In this work, we describe characterization of key microfluidic operations (droplet motion and splitting) underlying the EHP on the International Space Station (ISS). The testing was performed under the Advanced Passive Thermal eXperiment (APTx) project, a project to test a suite of passive thermal control devices funded by the ISS Technology Demonstration Office at NASA JSC.

A rapid manufacturing method was used to fabricate the electrowetting device on a printed circuit board. Key devicerelated considerations were to ensure reliability and package the experimental hardware within a confined space. Onboard the ISS, experiments were conducted to study electrowetting-based droplet motion and droplet splitting, by imaging droplet manipulation operations via pre-programmed electrical actuation sequences. An applied electric field of $36 \mathrm{Volts} / \mu \mathrm{m}$ resulted in droplet speeds approaching $10 \mathrm{~mm} / \mathrm{s}$. Droplet splitting dynamics was observed and the time required to split droplets was quantified. Droplet motion data was analyzed to estimate the contact line friction coefficient. Overall, this demonstration is the first-ever electrowetting experiment in space. The obtained results are useful for future design of the EHP and other electrowetting-based systems for microgravity applications.

\section{NOMENCLATURE}

$\begin{array}{ll}\text { A } & \text { Area, } m^{2} \\ \mathrm{C} & \text { Drag coefficient } \\ \mathrm{d} & \text { Plate spacing, } m m \\ \mathrm{~d}_{1} & \text { Thickness of bottom plate dielectric, } \mathrm{mm} \\ \mathrm{k}_{1} & \text { Dielectric constant } \\ \mathrm{m} & \text { Mass, } \mathrm{kg} \\ \mathrm{r} & \text { Radius, } m m \\ \mathrm{t} & \text { Time, } s \\ \mathrm{~V}_{1} & \text { Voltage, } V \\ \mathrm{v} & \text { Velocity, } \mathrm{mm} / \mathrm{s} \\ \mathrm{x} & \text { Displacement, } \mathrm{mm} \\ \varepsilon_{0} & \text { Permittivity of vacuum, } \mathrm{F} / \mathrm{m} \\ \mu_{1} & \text { Viscosity of droplet, } N \mathrm{~s} / \mathrm{m}^{2} \\ \rho_{\mathrm{f}} & \text { Filler fluid density, } \mathrm{g} / \mathrm{cm}^{3} \\ \zeta & \text { Friction coefficient, } N \mathrm{~s} / \mathrm{m}^{2}\end{array}$

\footnotetext{
*Corresponding author: enakshi.wik@ gmail.com
} 


\section{INTRODUCTION}

Heat pipes transport heat by absorbing heat at the evaporator (via evaporation of the working fluid) and rejecting it at the condenser (via condensation of working fluid). Heat pipes are closed-loop systems and conventional heat pipes use a wick to pump the condensate back from the condenser to the evaporator [1]. However, the condensate transport capacity of such a heat pipe is limited by the capillary pressure generated in the wick. Transporting heat over long distances is challenging since the fluid transport capacity (and thus heat transport capacity) of the wick varies inversely with length [2].

In an electrowetting heat pipe (EHP), the adiabatic wick section is replaced by electrical pumping of the condensate. Figure 1a shows the top view of an EHP [2,3], wherein the condensate is electrically pumped (as droplets) back to the evaporator in discrete channels. Separate vapor channels exist for the evaporated vapor to travel to the condenser. By replacing the wicking action with active electrical pumping, the capillary limit can be overcome. Preliminary estimates (based on related experiments) suggest that planar, water-based EHPs (with a 10 cm by $4 \mathrm{~mm}$ cross section) can transport more than $1.5 \mathrm{~kW}$ over extended distances $(>1 \mathrm{~m})$, with a thermal resistance of $0.01 \mathrm{~K} / \mathrm{W}$ $[2,3]$. Other advantages of the EHP include its compact nature, high turndown ratio, low weight, planar form factor, low power consumption and the absence of mechanical parts.

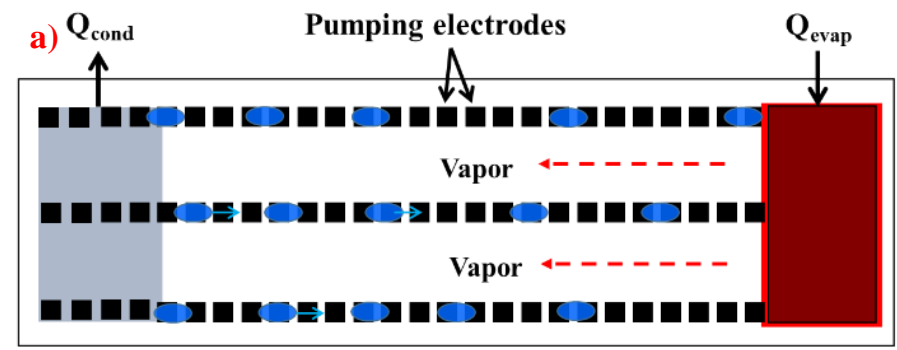

Condenser

Evaporator

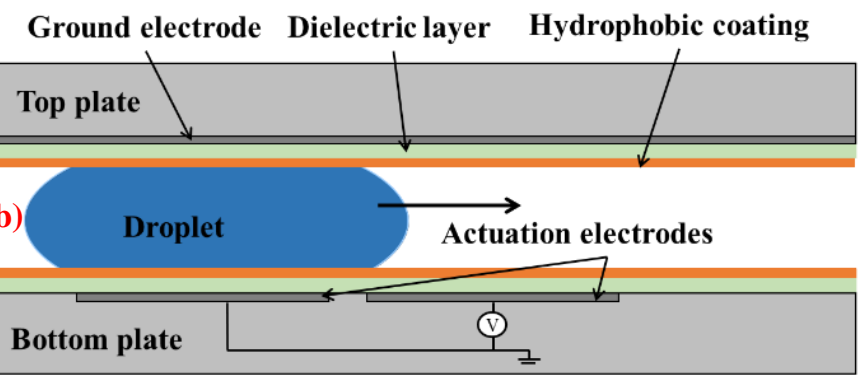

Figure 1. a) Schematic top view of an EHP, showing the droplet and vapor channels, and b) cross-section view of a two-plate device for EW-induced movement of droplets.

Such EHPs have applications in many systems where large heat loads need to be transported with low power consumption. EHPs can be used in applications related to power plant cooling, data center thermal management, and thermal management of slender tools and instrumentation associated with oil-gas drilling. The EHP also has space-relevant applications such as spacecraft thermal management and precision temperature control (normally accomplished using variable conductance heat pipes). Loop heat pipes are currently being used for space applications, but the EHP has distinct benefits over such heat pipes [2].

Electrical pumping in the EHP is achieved by using a microfluidic technology known as electrowetting (EW). EW is of great interest to the microfluidic community; applications being explored include lab-on-chip devices, displays, optical lenses, boiling heat transfer enhancement, and thermal management[4-7]. EW is based [4] on an effective reduction in the solid-liquid interfacial energy by applying a voltage difference between an electrically conducting droplet resting on a dielectric, and an underlying ground electrode. EW can enable various microfluidic operations such as moving, creating, mixing, and splitting droplets.

Most studies on EW actuation analyze EW-controlled droplet motion between two parallel plates [8,9], as shown in Figure 1b. Droplet motion is achieved by sequentially firing individually addressable control electrodes on the bottom plate while the top plate biases the droplet. Droplets follow the path of the traveling electric field. Hale et al. [3] conducted an experimental study on the microfluidic operations that would determine the performance of the EHP. Microfluidic operations studied included droplet motion, droplet splitting and the creation of droplets from an open reservoir (simulating the condensate pool). The objective was to understand the basic physics underlying fluid motion in an EHP, and estimate the maximum droplet volume that can be reliably pumped via EW.

While there are numerous studies on EW-induced droplet motion, there are no reported studies of EW in a microgravity environment. While the EW force is expected to exceed gravityrelated forces at the electric fields typically used for EW, the role of gravity on parameters such as contact line friction and hysteresis has not been studied.

The objective of this work is a study of the basic microfluidic operations (droplet pumping, splitting) underlying the EHP in a microgravity environment on the International Space Station (ISS). This is also the first reported EW experiment in space. It is noted that EW has other microgravity-related applications (in addition to the EHP).

\section{DEVICE FABRICATION AND EXPERIMENTAL PROCEDURE}

A microfluidic device was built and packaged using scalable manufacturing procedures. Since the target was to conduct microfluidics-related experiments only, heat transfer considerations were excluded while designing the device.

A printed circuit board (PCB) was used as the bottom plate. Electrode patterns were defined such as to enable droplet motion and splitting experiments. The electrode pattern consisted of two arrays of ten electrodes, with each electrode having dimensions $2 \mathrm{~mm} \times 2 \mathrm{~mm}$. The spacing between two arrays on the PCB was $0.17 \mathrm{~mm}$. The spacing between the PCB and the top plate was determined by the thickness of a stainless steel shim stock 
between the two plates. The PCBs with the etched electrode patterns were fabricated by a commercial vendor, Express PCB. The PCBs were sanded to expose the copper layer and ensure that the area over which the droplet moves is smooth and uniform.

A $12.5 \mu \mathrm{m}$ thick polyimide layer (Kapton tape) was used as the EW dielectric. The EW dielectric is key to the operation of the device. Thin dielectrics $(\sim 1 \mu \mathrm{m})$ are ideal, since the required voltages can be reduced. However many thin dielectrics like Parylene $\mathrm{C}$ have pinholes and other defects, which can severely compromise the reliability of the device. A thicker dielectric was therefore selected for device reliability reasons, even though it increased the operating voltage by a factor of $\sim 10$.

The device was cleaned with acetone and isopropanol after applying the dielectric. A $1 \mu \mathrm{m}$ layer of CYTOP (hydrophobic polymer) layer was then spin-coated on the surface. Hydrophobicity reduces contact angle hysteresis, and reduces the required voltage. Post CYTOP deposition, the surface was soft baked at $120^{\circ} \mathrm{C}$ for 15 minutes and then hard baked at $180^{\circ} \mathrm{C}$ for 1 hour. After the board cooled down, it was immersed in a silicone oil bath for at least 24 hours. The absorption of silicone oil at the surface increases hydrophobicity and lubrication, and makes droplet transport more reliable and repeatable.

The top plate consisted of a $2 \mathrm{~cm} \times 2 \mathrm{~cm}$ polycarbonate plate coated with a thin layer of Indium Tin Oxide (ITO). ITO is an electrically conducting and transparent oxide. The top plate was spin coated with a $1 \mu \mathrm{m}$ layer of Cytop following the same procedure used for the bottom plate. The top plates were also soaked in a silicone oil bath for 24 hours.

The devices were assembled using nylon and rubber screws, bolts and washers. Stainless steel shim-stocks of thickness 0.127 $\mathrm{mm}$ and $0.254 \mathrm{~mm}$ were used as spacers between the bottom and top plates; these spacers decided the gap between the plates. They were also used to electrically ground the top plate. Figure 2 shows a completely assembled board, including the grounded top plate and stainless steel spacers. It is again emphasized that this procedure is scalable and non-cleanroom-based; larger/longer devices can be built using the techniques described.

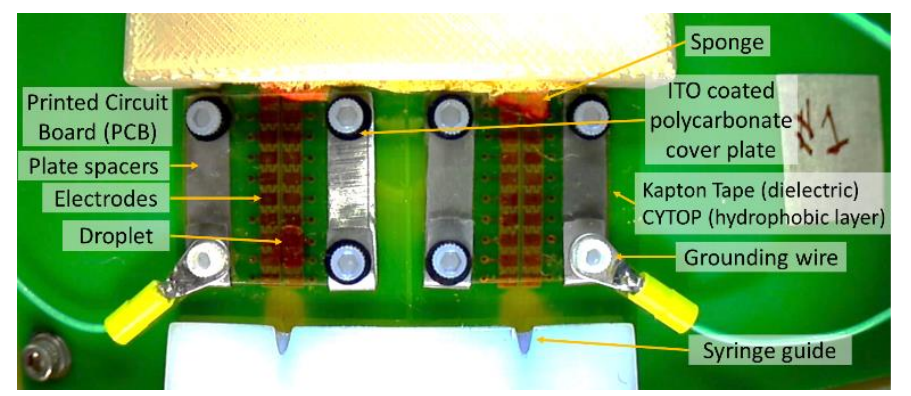

Figure 2. Top view of completely assembled EHP device for characterization.

The electrical circuitry to enable droplet motion and splitting is described next. Each electrode in the EHP array was independently actuated; the actuation sequence enabled droplet motion and splitting. To activate an electrode, a digital input signal of " 1 " was entered. Next, an optocoupler connected this low power signal to a $5 \mathrm{~V}$ source, providing the amplifying current for a bipolar junction transistor (BJT). The BJT coupled the electrode to the high voltage source, set to $450 \mathrm{~V}$ to effectively set the electrode at $450 \mathrm{~V}$. Each electrode was brought back down to $0 \mathrm{~V}$ by entering a logical " 0 " to the circuit.

The microfluidic device was packaged and sent to NASA Johnson Space Center near Houston TX. It was subjected to various compliance and safety-related checks before it was flown to the ISS. The device was unpackaged on the ISS and experiments were conducted subsequently in two rounds.

Droplet movement sequences were pre-programed on the ground and controlled from NASA Johnson Space Center. These programs consisted of various actuation patterns to trigger droplet motion and splitting and are described in a previous article [3]. Droplets were manually dispensed at the upstream end of the device by the crew of the ISS. Droplet motion was recorded at 30 frames per second using a camera with a fish-eye lens, with live video streamed into the control room.

\section{RESULTS}

All experiments on droplet motion and splitting were conducted at $450 \mathrm{~V}$; the high voltages are primarily a consequence of using a very thick dielectric layer. Images of droplet motion and splitting were used to extract data on droplet speeds and the time required for splitting.

Figure 3 illustrates droplet motion, based on videos taken on the ISS. The two sequences correspond to plate spacings of 0.254 $\mathrm{mm}$ and $0.127 \mathrm{~mm}$, respectively. The liquid slug volume in both cases was $1 \mu \mathrm{l}$ and the electrodes were switched at a frequency of 1 second. Image analysis was used to estimate the droplet velocity as it moved along the electrode array. The velocity was determined by measuring the distance moved by the leading edge of the droplet for a given time. It is noted that the leading and trailing edges move at different speeds, however only the 'average' droplet speed is measured in these experiments.

a)
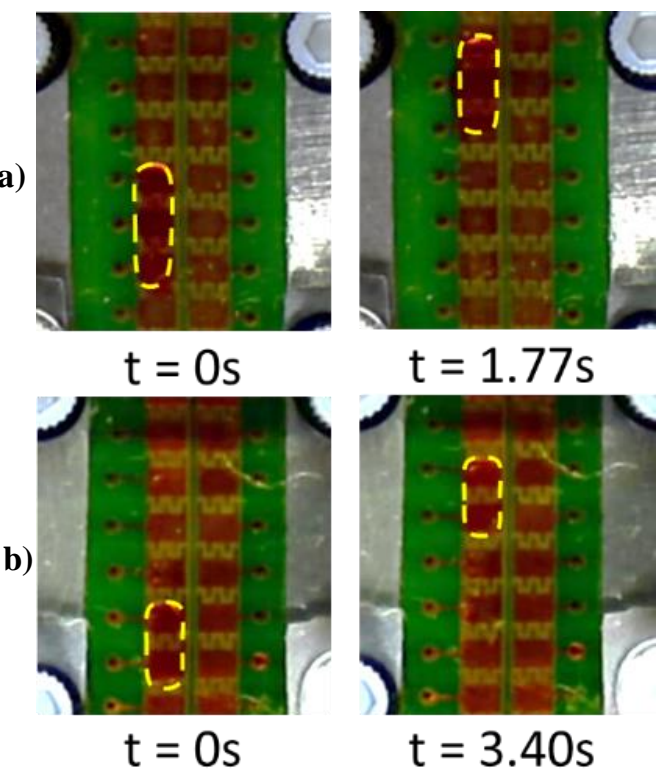
Figure 3. Images showing droplet motion under a $36 \mathrm{~V} / \mathrm{micron}$ field for a channel gap of (a) $0.254 \mathrm{~mm}$ and (b) $0.127 \mathrm{~mm}$. Droplet shape is highlighted by the dotted line for clarity.

Droplet velocity versus volume data is shown in Figure 4 and was estimated by averaging the velocities of the droplet as it moved once along the array of electrodes as per the programed sequence. The velocities at the beginning and end of the motion on an array was excluded, to eliminate entrance and exit-related effects.

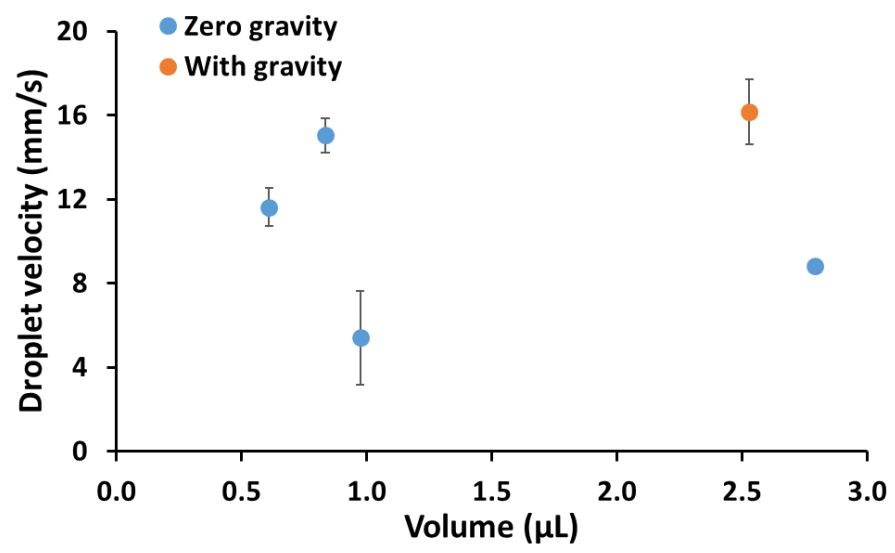

Figure 4. Experimental measurements of droplet velocities under EW actuation using $450 \mathrm{~V}$.

Figure 4 shows that droplets velocities of upto $15 \mathrm{~mm} / \mathrm{sec}$ were attained at $450 \mathrm{~V}$, this corresponds to an electric field of 36 $\mathrm{V} /$ micron. Figure 4 also shows significant scatter in the velocity measurements. Comparison with droplet speeds in the lab indicates that the droplets moved faster by $7 \%-75 \%$ in the lab as compared to under microgravity. These discrepancies could be a result of surface degradation with time, contamination and possible circuitry-related issues.

Similar experiments were also conducted to demonstrate splitting of droplets. Splitting is achieved by electrically actuating the droplets to move in opposite directions at the same time [3], this causes the droplet to be pulled in two directions, which leads to splitting at the center. In the present experiments, splitting was accomplished by spreading the droplet along three activated electrodes, and deactivating the middle electrode to induce necking. Adjacent electrodes were then activated to pull the droplet apart. Figure 5 illustrates droplet splitting sequences for plate spacings of $0.254 \mathrm{~mm}$ (Fig. 5a) and $0.127 \mathrm{~mm}$ (Fig. 5b).
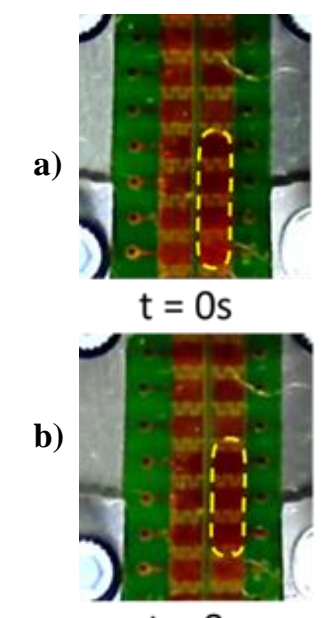

$\mathrm{t}=0 \mathrm{~s}$

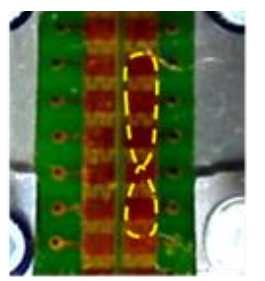

$\mathrm{t}=1.3 \mathrm{~s}$

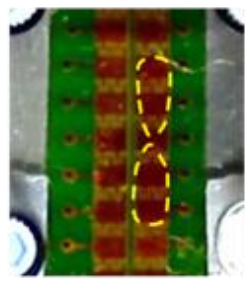

$\mathrm{t}=1.70 \mathrm{~s}$

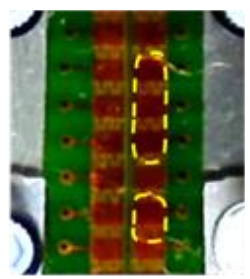

$\mathrm{t}=1.53 \mathrm{~s}$

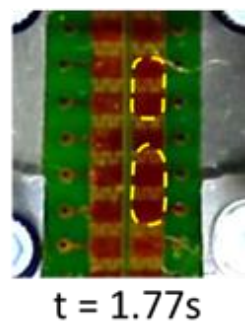

Figure 5. Images showing droplet splitting under a $36 \mathrm{~V} / \mathrm{micron}$ field for a channel gap of (a) $0.254 \mathrm{~mm}$ and (b) $0.127 \mathrm{~mm}$. Droplet shape is highlighted by the dotted line for clarity.

Image analysis was used to quantify the time required for splitting droplets. Droplet splitting was defined as the time interval starting from when the droplet leading edge began to move to the time that the droplet completely separated into two. For comparison purposes a split ratio was defined as:

$$
\text { Split Ratio }=\frac{\text { Seperated volume }}{\text { Initial volume }}
$$

where the separated volume is the portion of the droplet that continues to move up the array of electrodes after splitting, as shown in Figure 5. The initial volume is the total droplet volume prior to splitting.

Figure 6 shows all experimentally measured splitting times obtained from videos at $450 \mathrm{~V}$ operation. An average splitting time of $1.5 \pm 0.6$ seconds was found based on all available data. Studies using a similar set up in the lab show splitting times of $1.07 \pm 0.05 \mathrm{~s}[3]$. The higher splitting times observed on the ISS could be due to surface degradation over time.

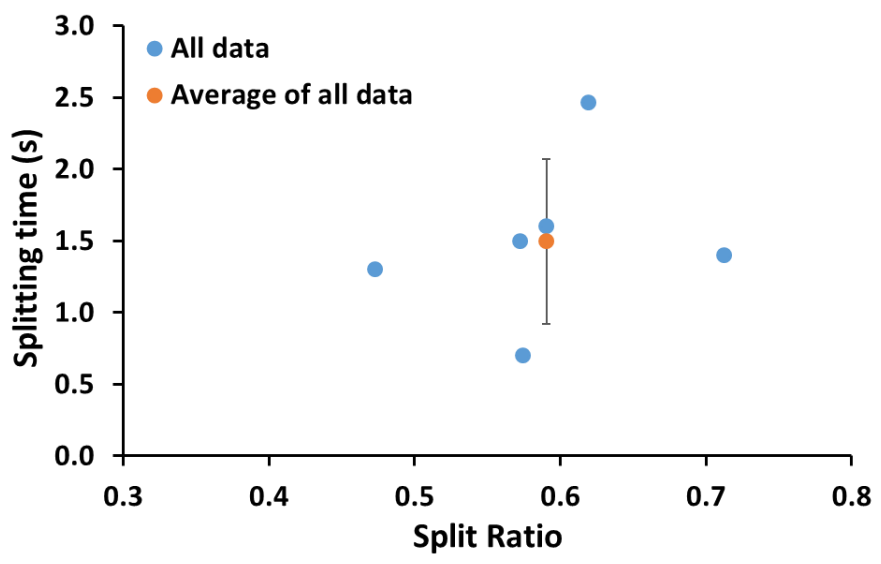

Figure 6. Experimental measurements of the time required to split a droplet using EW actuation of $450 \mathrm{~V}$. 


\section{ANALYSIS}

The droplet motion experiments were used to estimate the contact line friction associated with droplet motion. This was directly compared to data from the lab. The contact line friction coefficient was calculated based on a previously developed model of EW actuation by Bahadur et al [8]. The model simulates an EW-induced droplet motion which is opposed by shear forces from the top and bottom plates, viscous forces due to the surrounding media and contact-line friction. The force balance can be represented as:

$$
m \frac{d^{2} x}{d t^{2}}=F_{\text {actuation }}-F_{w}-F_{f}-F_{c l}
$$

The electrowetting actuation force can be expressed as [8]:

$$
F_{\text {actuation }}=\frac{k_{1} \varepsilon_{0} V^{2}}{2 d_{1}} \frac{d A}{d x}
$$

The shear force from the top and bottom plates is modeled by assuming a parabolic velocity profile of the droplet between the two plates $[8,10]$ as:

$$
F_{w}=\left(\frac{6 \mu_{l} v}{d}\right)\left(2 \pi r^{2}\right)
$$

The shear force due to the surrounding media (air) is derived by assuming that the droplet moves as a rigid body [8]:

$$
F_{f}=\left(\frac{1}{2} C \rho V^{2}\right)(2 r d)
$$

The contact line friction force is modeled to be linearly proportional to the contact line velocity as [8]:

$$
F_{c l}=(\zeta v)(4 \pi r)
$$

Here, $\zeta$ is the friction coefficient or a proportionality coefficient between the friction force and droplet velocity [8, 11]. Fundamentally, the contact line friction force originates from intermolecular attraction forces and surface roughness.

An average value of the contact line friction coefficient was calculated based on the range of velocities shown in Figure 4. The average friction coefficient under microgravity was calculated to be $2.7 \pm 1.4 \mathrm{Ns} / \mathrm{m}^{2}$. Similar estimates using data from the lab result in a friction coefficient of $1.9 \pm 0.3 \mathrm{Ns} / \mathrm{m}^{2}$. The results suggest that contact line friction under microgravity is

\section{REFERENCES}

[1] A. Faghri, 1995 "Heat pipe science and technology," Taylor \& Francis.

[2] R. S. Hale and V. Bahadur, 2015, "Electrowetting Heat Pipes for Heat Transport over Extended Distances," IEEE Trans. Components, Packag. Manuf. Technol., 5(10), pp. 1441-1450.

[3] R. S. Hale and V. Bahadur, 2017, "Electrowetting-based microfluidic operations on rapid-manufactured devices for heat pipe applications," J. Micromechanics Microengineering, 27(7).

[4] F. Mugele and J.-C. Baret, 2005, "Electrowetting: from basics to applications," J. Phys. Condens. Matter, 17(28), pp. R705-R774.

[5] A. Shahriari, P. Birbarah, J. Oh, N. Miljkovic, and V. Bahadur, 2017, "Electric Field-Based Control and Enhancement of Boiling and Condensation," Nanoscale higher under microgravity than under terrestrial conditions. However, the error bars associated with these measurements are high, which indicates that the results must be interpreted cautiously.

Previously reported values of the contact line friction for similar systems are as low as $0.04 \mathrm{Ns} / \mathrm{m}^{2}[8,11]$. The contact line friction model used is accurate for higher velocities than those in the current experiments; this partly explain the discrepancies. However, all the experimental results together suggest that droplets in the current experiments required larger actuation forces than expected. A primary reason for this could be surface degradation and loss of silicone oil over the nine-month period from the final assembly of the device to actual experimentation.

\section{CONCLUSIONS}

In summary, the present work reports the first demonstration of EW technology in space. The present experiments show that EW experiments can be safely conducted in space, and that any safety, reliability and compactness-related challenges can be overcome. This work complements an existing study on microfluidic operations on earth, and shows that the microfluidic performance under microgravity will be broadly comparable to that on land. Future research is targeted at thermal characterization of such heat pipes. It should also be noted that EW can be the basis of other microfluidic technologies that can benefit space research and applications.

\section{ACKNOWLEDGEMENTS}

This effort was organized by the NASA Johnson Space Center Passive Thermal Technical Discipline Lead, the Exploration Technology Office and funded by the ISS Technology Demonstration Office. The contributions of several personnel at NASA Johnson Space Center and the crew of the ISS are acknowledged. Also, acknowledged is the effort of the support staff at UT Austin.

Microscale Thermophys. Eng., 21(2), pp. 102-121.

[6] S. Haeberle and R. Zengerle, 2007, "Microfluidic platforms for lab-on-a-chip applications," Lab Chip, 7(9), pp. 1094-1110.

[7] S.-Y. Park, J. Cheng, and C.-L. Chen, 2012, "Active Hot Spot Cooling Controlled by Single-Sided Electrowetting-on-Dielectric (SEWOD), " in Proceedings of the ASME 2012 Summer Heat Transfer Conference, pp. 617-623.

[8] V. Bahadur and S. V. Garimella, 2006, "An energy-based model for electrowetting-induced droplet actuation," J. Micromechanics Microengineering, 6, pp. 1494-1503.

[9] N. Kumari, V. Bahadur, and S. V. Garimella, 2008, "Electrical actuation of electrically conducting and insulating droplets using ac and dc voltages," J. Micromechanics Microengineering, 18(10).

[10] Jason S. Kuo, Paolo Spicar-Mihalic, A. Indalesio 
Rodriguez, and D. T. Chiu*, 2002, "ElectrowettingInduced Droplet Movement in an Immiscible Medium," Langmuir, 19(2), pp. 250-255.

[11] H. Ren, R. B. Fair, M. G. Pollack, and E. J. Shaughnessy,
2002, "Dynamics of electro-wetting droplet transport," Sensors Actuators B Chem., 87(1), pp. 201-206. 